\title{
Bounds for Minimum Feedback Vertex Sets in Distance Graphs and Circulant Graphs
}

\author{
Hamamache Kheddouci ${ }^{1}$ and Olivier Togni ${ }^{2}$ \\ ${ }^{1}$ LIESP, Université Claude Bernard Lyon1, 843, Bd. du 11 novembre 1918, 69622 Villeurbanne Cedex France, \\ hkheddoulbat 710 .univ-lyon1.fr \\ ${ }^{2}$ LE2I, UMR CNRS 5158, Université de Bourgogne, BP 47870, 21078 Dijon Cedex, France \\ olivier.togni@u-bourgogne.fr
}

received april 10, 2006, revised july 25, 2007, accepted february 11, 2008.

For a set $D \subset \mathbb{Z}_{n}$, the distance graph $P_{n}(D)$ has $\mathbb{Z}_{n}$ as its vertex set and the edges are between vertices $i$ and $j$ with $|i-j| \in D$. The circulant graph $C_{n}(D)$ is defined analogously by considering operations modulo $n$. The minimum feedback vertex set problem consists in finding the smallest number of vertices to be removed in order to cut all cycles in the graph. This paper studies the minimum feedback vertex set problem for some families of distance graphs and circulant graphs depending on the value of $D$.

Keywords: Feedback Vertex Set, Distance graph, Circulant graph.

\section{Introduction}

For a finite undirected graph $G=(V, E)$, the minimum feedback vertex set problem is to find a set of vertices in $G$ of minimum cardinality whose removal induces a forest. We shall denote by $\bar{V}$ a Feedback Vertex Set (FVS for short) and by $F(G)$ the minimum of the cardinalities over all the FVS: $F(G)=$ $\min \{|\bar{V}|, \bar{V}$ is a FVS $\}$. A minimum feedback vertex set (MFVS) is a FVS of cardinality $F(G)$.

This parameter has received much attention since finding a MFVS of a graph has a lot of applications in various areas such as combinatorial circuit design, deadlock prevention in computer systems, VLSI testing, artificial intelligence [BG94] and converter placement in optical networks [Tog00].

The MFVS problem is known to be NP-complete for general graphs [GJ79]. For undirected graphs, the best known algorithm found in [BG94] and [BBF99] is a 2-approximation. A lot of work has also been done on finding exact values for specific graphs such as hypercubes [FLP00, Pik03], meshes and butterflies [Luc98, CKK02, PK05], pyramids and mesh of trees [Luc03]. Recently, an interesting connection was made in [FGR02] between the MFVS problem and the acyclic coloring problem, which allows the authors to derive bounds for a number of families of graphs such as graphs of maximum degree 3 and 4 , planar graphs and $k$-trees. The reader is referred to [FPR99] for a rather complete survey on feedback sets.

In this paper, we study the MFVS problem on finite distance graphs and circulant graphs. 
The (infinite) distance graph $P(D)$ with distance set $D=\left\{d_{1}, d_{2}, \ldots, d_{k}\right\}$, where $d_{i}$ being positive integers, has the set $\mathbb{N}$ of integers as vertex set, with two distinct vertices $i, j \in \mathbb{N}$ being adjacent if and only if $|i-j| \in D$. The finite distance graph $P_{n}(D)$ is the subgraph of $P(D)$ induced by vertices $0,1, \ldots, n-1$. Remark that there are $n-d_{i}$ chords of length $d_{i} \leq n$ in $P_{n}(D)$. Thus the number of edges of $P_{n}(D)$ is $|E|=k n-\sum_{i=1}^{k} d_{i}$. The study of distance graphs was initiated by Eggleton et al. [EES85]. A large amount of work has focused on colorings of distance graphs [EES90, Voi99, EK03].

The circulant graph can be defined as follows: let $n$ be a natural number and let $D=\left\{d_{1}, d_{2}, \ldots, d_{k}\right\}$, with $1 \leq d_{1}<d_{2}<\ldots<d_{k} \leq n / 2$. Then the vertex set of the circulant graph $C_{n}(D)$ is $\{0,1, \ldots, n-$ $1\}$ and the set of neighbors of the vertex $i$ is $\left\{\left(i \pm d_{j}\right) \bmod n, j=1,2 \ldots, k\right\}$. Circulant graphs (also called loop networks) have been widely studied for their interesting properties in terms of diameter, symmetry and connectivity. A survey paper about loop networks is given in [BCH95].

To simplify, we will sometimes omit the brackets thus writing $P_{n}\left(d_{1}, d_{2}, \ldots, d_{k}\right)\left(C_{n}\left(d_{1}, d_{2}, \ldots, d_{k}\right)\right.$, respectively) instead of $P_{n}\left(\left\{d_{1}, d_{2}, \ldots, d_{k}\right\}\right)\left(C_{n}\left(\left\{d_{1}, d_{2}, \ldots, d_{k}\right\}\right)\right.$, respectively).

Notice that the distance graph $P_{n}(D)$ is a subgraph of the circulant graph $C_{n}(D)$. Using this fact, we will first find feedback vertex sets for finite distance graphs $P_{n}(D)$, for different values of $D$ in Section 2 and then transform these results to find feedback vertex sets for circulant graphs $C_{n}(D)$ with the same sets $D$ in Section 3 .

Our main results are summarized in Table 1.

\begin{tabular}{|c|c|c|c|c|}
\hline & \multicolumn{2}{|c|}{$F\left(P_{n}(D)\right)$} & \multicolumn{2}{|c|}{$F\left(C_{n}(D)\right)$} \\
\hline$D$ & Lower bound & Upper bound & Lower bo & Upper bound \\
\hline$\{1,2\}$ & \multicolumn{2}{|c|}{$\left\lceil\frac{n-2}{3}\right\rceil$} & \multicolumn{2}{|c|}{$\left\lfloor\frac{n+4}{3}\right\rfloor$} \\
\hline$\{1, t\}$ & $\left\lceil\frac{n-t}{3}\right\rceil$ & $\left\lceil\frac{n-2}{3}\right\rceil^{*}$ & $\left\lceil\frac{n+1}{3}\right\rceil$ & $\left\lceil\frac{n+t}{3}\right\rceil+1$ \\
\hline$\{1,2,4\}$ & $4\left\lfloor\frac{n}{8}\right\rfloor$ & $\left\lceil\frac{n}{2}\right\rceil$ & $4\left\lfloor\frac{n}{8}\right\rfloor$ & $\left\lceil\frac{n+4}{2}\right\rceil$ \\
\hline$\{1, s, t\}$ & $\left\lceil\frac{2 n-s-t}{5}\right\rceil$ & $\left.\frac{n}{2}\right\rceil$ & $\frac{2 n+1}{5}$ & $\left\lceil\frac{n+t}{2}\right\rceil$ \\
\hline$\{1,2,3, \ldots, t\}$ & \multicolumn{2}{|c|}{$\begin{array}{l}\left\lfloor\frac{n}{t+1}\right\rfloor(t-1), \text { if } n \equiv 0,1 \bmod (t+1) \\
n-2\left\lfloor\frac{n}{t+1}\right\rfloor-2, \text { otherwise }\end{array}$} & $n-2\left\lfloor\frac{n}{t+1}\right\rfloor-1^{* *}$ & $n-2\left\lfloor\frac{n}{t+1}\right\rfloor^{* *}$ \\
\hline
\end{tabular}

Tab. 1: Bounds for the MFVS of distance graphs and circulant graphs

Before presenting our results, let us remember a lower bound found in [CKK02] that will be used all along this paper:

Lemma 1 ([|CKK02]) Let $G$ be a graph of order $n$ and size $m$, with maximum degree $\Delta$. Then

$$
F(G) \geq\left\lceil\frac{m-n+1}{\Delta-1}\right\rceil
$$

Proof: Let $G^{\prime}$ be a graph obtained from $G$ by removing $f$ vertices and all edges incident to them. The minimum number of edges $m^{\prime}$ of $G^{\prime}$ satisfies $m^{\prime} \geq m-\Delta f$ and the order of $G^{\prime}$ is $n^{\prime}=n-f$. The 
graph $G^{\prime}$ cannot be acyclic if $m^{\prime} \geq n^{\prime}$ i.e. if $f<\left\lceil\frac{m-n+1}{\Delta-1}\right\rceil$. Thus any FVS must have at least $\left\lceil\frac{m-n+1}{\Delta-1}\right\rceil$ vertices.

\section{Feedback vertex sets of distance graphs}

\section{$2.1 D=\{1, t\}$}

Proposition 1 For any integer $t \geq 2$,

$$
F\left(P_{n}(1, t)\right)= \begin{cases}0 & \text { if } n \leq t, \\ \left\lceil\frac{n-t}{2}\right\rceil & \text { if } \frac{n}{2} \leq t<n, \\ \left\lfloor\frac{n+2}{4}\right\rfloor & \text { if } \frac{n}{3} \leq t<\frac{n}{2} .\end{cases}
$$

Proof: Let $G=P_{n}(1, t)$. If $t \geq n$ then $G$ is a path.

If $\frac{n}{2} \leq t<n$ then the maximum degree of $G$ is 2 if $n=t+1$ and 3 otherwise. $P_{t+1}(1, t)$ is a cycle, thus $F\left(P_{t+1}(1, t)\right)=1=\left\lceil\frac{1}{2}\right\rceil$. For $n \geq t+2$, applying Lemma 1 with $m=2 n-t-1$ gives $F\left(P_{n}(1, t)\right) \geq\left\lceil\frac{n-t}{2}\right\rceil$. On the other hand, we construct a FVS $\bar{V}$ in this way: we skip the $t$ first vertices and we pick alternately one on two of the $n-t$ last vertices (see Figure 1). More precisely, $\bar{V}=\left\{t+2 i, 0 \leq i<\left\lceil\frac{n-t}{2}\right\rceil\right\}$.

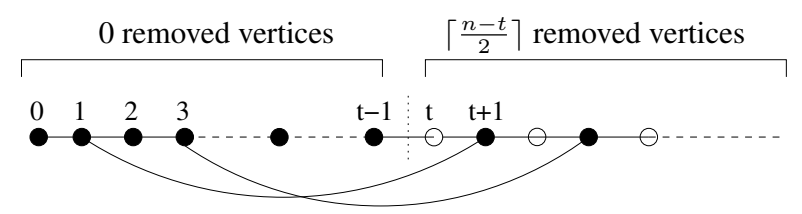

Fig. 1: Structure of the FVS $\bar{V}$ (non filled circles represent vertices in the FVS).

Note that the $t$ first vertices of $G$ do not induce any cycle and the $n-t$ remaining vertices have degree at most 1 in $G \backslash \bar{V}$. So $\bar{V}$ is a FVS.

If $\frac{n}{3} \leq t<\frac{n}{2}$, let $n_{i}$ be the number of vertices of degree $i$ in $G$. It is clear that the first and the last vertices of $G$ are of degree two, so $n_{2}=2$. Moreover, vertices $1,2, \ldots t-1$ and $n-t, n-t+1, \ldots, n-2$ are of degree 3 , thus $n_{3}=2 t-2$. Therefore, $n_{4}=n-n_{3}-n_{2}=n-2 t$.

Let $\bar{V}$ be a MFVS of $G$. Assume that the vertices of $\bar{V}$ are removed from $G$ one by one in a certain order. Suppose that $|\bar{V}|=\alpha+\beta$, where $\alpha$ is the number of vertices that were of degree 4 just before they were removed (i.e. each removed vertex contributes to decrease the number of edges by 4 ) and $\beta$ is the number of vertices that were of degree less than 4 just before they were removed (i.e. each removed vertex contributes to decrease the number of edges by 3 or less). Notice that these $\alpha$ vertices are of degree 4 in $G$ and form an independent set. There are $n_{4}=n-2 t$ vertices of degree 4 in $G$ that are consecutive on the graph, thus $\alpha \leq\left\lceil\frac{n-2 t}{2}\right\rceil$.

Let $n^{\prime}$ ( $m^{\prime}$, respectively) be the number of vertices (edges, respectively) of $G^{\prime}=G \backslash \bar{V}$. We have $n^{\prime}=n-\alpha-\beta$ and $m^{\prime} \geq|E(G)|-4 \alpha-3 \beta=2 n-t-1-4 \alpha-3 \beta$. As $G^{\prime}$ is a forest, we have 
$m^{\prime} \leq n^{\prime}-1$ and thus $2 n-t-1-4 \alpha-3 \beta \leq n-\alpha-\beta-1$ or equivalently,

$$
3 \alpha+2 \beta \geq n-t
$$

As $\bar{V}$ is a MFVS, we want $\alpha+\beta$ to be minimized subject to the above constraints. In order to do so we must maximize $\alpha$. Therefore the solution is given by setting $\alpha=\left\lceil\frac{n-2 t}{2}\right\rceil$ and $\beta=\left\lceil\frac{n-t-3 \alpha}{2}\right\rceil$. Thus $F(G) \geq\left\lceil\frac{n-2 t}{2}\right\rceil+\left\lceil\frac{n-t-3\left\lceil\frac{n-2 t}{2}\right\rceil}{2}\right\rceil=\left\lfloor\frac{n+2}{4}\right\rfloor$.

Now, we have to construct a FVS of cardinality $\left\lfloor\frac{n+2}{4}\right\rfloor$ : we begin, as for the case $t<n \leq 2 t$, by skipping the $t$ first vertices and picking alternately one on two of the next $t$ vertices. For the $n-2 t$ remaining vertices, if $t$ is odd then we skip three vertices and pick one and so on for the last $n-2 t$ vertices (see Part $a$ ) of Figure 2) and if $t$ is even, we skip one vertex then pick one, then skip three and pick one repetitively until the end (see Part $b$ ) of Figure 2).

More precisely, $\bar{V}=\left\{2 i+t, 0 \leq i \leq \frac{t-1}{2}\right\} \cup\left\{2 t+3+4 i, 0 \leq i \leq\left\lfloor\frac{n-2 t-4}{4}\right\rfloor\right\}$ for $t$ odd and $\bar{V}=\left\{2 i+t, 0 \leq i \leq \frac{t-2}{2}\right\} \cup\left\{2 t+1+4 i, 0 \leq i \leq\left\lfloor\frac{n-2 t-2}{4}\right\rfloor\right\}$ for $t$ even.

Thus $|\bar{V}|=\frac{t-1}{2}+1+\left\lfloor\frac{n-2 t-4}{4}\right\rfloor+1=\left\lfloor\frac{n+2}{4}\right\rfloor$ for odd $t$ and $|\bar{V}|=\frac{t-2}{2}+1+\left\lfloor\frac{n-2 t-2}{4}\right\rfloor+1=\left\lfloor\frac{n+2}{4}\right\rfloor$ for even $t$.
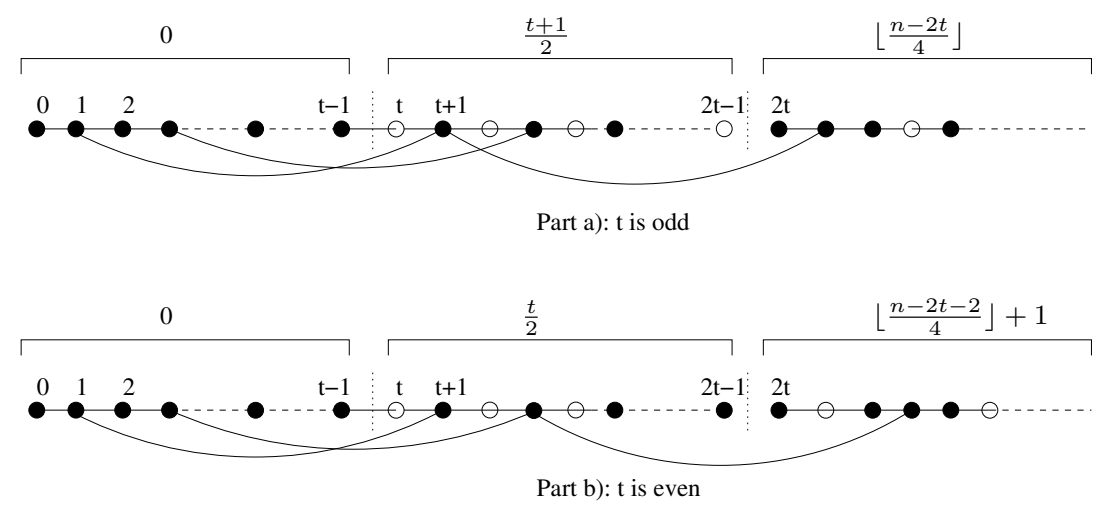

Fig. 2: Structure of the FVS $\bar{V}$ (non filled circles represent vertices in the FVS).

The set $\bar{V}$ is a FVS because the first block of $t$ vertices does not induce any cycle in $G^{\prime}=G \backslash \bar{V}$, vertices of the second block of $t$ vertices are of degree 2 in $G^{\prime}$ and the third block of $n-2 t$ vertices induces disjoint subgraphs $P_{3}$. Moreover, each $P_{3}$ consists in two vertices of even indices and one vertex of odd index and each vertex of the second block has the same parity as $(t+1)$. Hence, only the vertex of odd index of each $P_{3}$ is linked by a chord with a vertex of the second block.

Proposition 2 For any $n$ and any $t$, with $t \geq 2$ and $n \geq t$,

$$
\left\lceil\frac{n-t}{3}\right\rceil \leq F\left(P_{n}(1, t)\right) \leq\left\lceil\frac{n-2}{3}\right\rceil .
$$


Proof: Let $G=P_{n}(1, t)$. The lower bound comes from Lemma 1 as $G$ is of order $n$, size $2 n-t-1$ and maximum degree at most 4 .

For the upper bound, we cut the proof in two parts:

Case 1: $t \not \equiv 0 \bmod 3$. Let $\bar{V}=\left\{3 j-1,1 \leq j \leq\left\lfloor\frac{n}{3}\right\rfloor\right\}$ be the vertex set which we remove from $G$. By contradiction, suppose that $G^{\prime}=G \backslash \bar{V}$ contains a cycle $C$.

Let $i$ be the vertex of $C$ of minimum index. Then vertex $i$ must be adjacent to vertices $i+1$ and $i+t$ in $C$. We have $i \not \equiv 2 \bmod 3$ and $i+1 \not \equiv 2 \bmod 3$ thus $i \equiv 0 \bmod 3$ and $i+2$ cannot be a vertex of $C$. Therefore, vertex $i+1$ must be adjacent to vertex $i+t+1$ in $C$ and thus $C$ is a cycle of order 4 (see Figure 3].

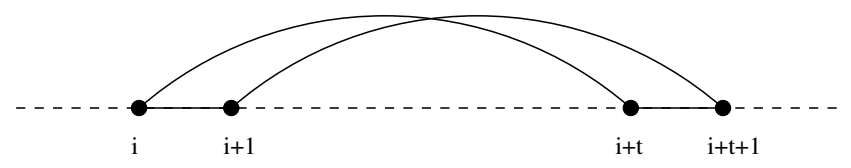

Fig. 3: The cycle $C$

As $t \not \equiv 0 \bmod 3$, it turns out that either $i+t \equiv 2 \bmod 3$ or $i+t+1 \equiv 2 \bmod 3$, which is a contradiction. Thus in that case, $\bar{V}$ is a FVS of $G$ of cardinality $|\bar{V}|=\left\lfloor\frac{n}{3}\right\rfloor=\left\lceil\frac{n-2}{3}\right\rceil$.

Case 2: $t \equiv 0 \bmod 3$. Let $q$ be the integer such that $t=3 q$. We are going to construct a FVS with a periodic pattern of period $2 t$ :

Let $\bar{V}=\{x \in \bar{W}, x \leq n-1\}$, with $\bar{W}=\bigcup_{j=0}^{\left\lceil\frac{n}{2 t}\right\rceil-1}\left(A_{j} \cup B_{j}\right)$, where

$A_{j}=\{2 t j+3 i, 1 \leq i \leq q\}$ and $B_{j}=\{2 t j+3(q+i)-1,1 \leq i \leq q\}$.

Figure 4 gives an example of such a set.

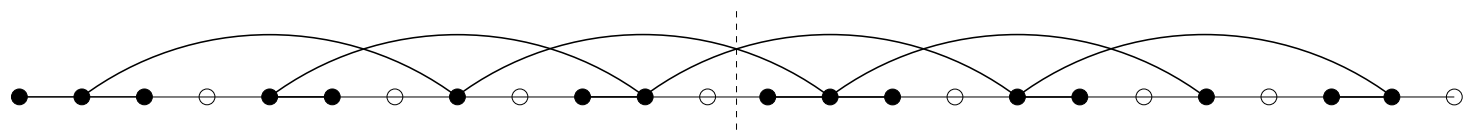

Fig. 4: FVS in $P_{24}(1,6)$ (non filled circles represent vertices in the FVS)

First observe that $A_{j} \cap B_{j}=\emptyset$ for any $j$. Next, to prove that $\bar{V}$ is a FVS, as for the previous case, suppose that $G \backslash \bar{V}$ contains a cycle $C$. Let $\alpha$ be the vertex of $C$ of minimum index. The neighbors of $\alpha$ on the cycle $C$ are $\alpha+1$ and $\alpha+t$; then the only cases to study are when $\alpha \notin \bar{V}, \alpha+1 \notin \bar{V}$ and $\alpha+t \notin \bar{V}$. If, for some $j, 0 \leq j \leq\left\lceil\frac{n}{2 t}\right\rceil-1$, the vertex $\alpha$ is of the form:

1. $\alpha=2 t j$ then $\alpha+t=2 t j+3 q \in \bar{V}$.

2. $\alpha=2 t j+3 i$ for some $i, 1 \leq i \leq q$ then $\alpha \in \bar{V}$.

3. $\alpha=2 t j+3 i+1$ for some $i, 0 \leq i \leq q-1$, then $\alpha+2=2 t j+3(i+1) \in \bar{V}$; the cycle $C$ must be $C=(\alpha, \alpha+1, \alpha+t+1, \alpha+t, \alpha)$. But $\alpha+t+1=2 t j+3(q+i)+2=2 t j+3(q+i+1)-1 \in \bar{V}$, a contradiction. 
4. $\alpha=2 t j+3 i+2$ for some $i, 0 \leq i \leq q-1$ then $\alpha+1=2 t j+3(i+1) \in \bar{V}$.

5. $\alpha=(2 j+1) t+3 i$ for some $i, 1 \leq i \leq q-1$ then $\alpha+t=2(j+1) t+3 i \in \bar{V}$.

6. $\alpha=(2 j+1) t+3 i+1$ for some $i, 0 \leq i \leq q-1$ then $\alpha+1=2 t j+3(q+i+1)-1 \in \bar{V}$.

7. $\alpha=(2 j+1) t+3 i+2$ for some $i, 0 \leq i \leq q-1$ then $\alpha \in \bar{V}$.

Now we have to show that $|\bar{V}| \leq\left\lfloor\frac{n}{3}\right\rfloor$. By definition, we have $\left|A_{j}\right|=\left|B_{j}\right|=q$. Thus, on a period of $2 t=6 q$ vertices, the feedback vertex set has cardinality $2 q=\frac{2 t}{3}$. It remains to see that for the last $k$ vertices of $G(k<2 t)$, at most $\left\lceil\frac{k-2}{3}\right\rceil$ of them are in $\bar{V}$. If $k \leq t$ then $\left\lceil\frac{k-3}{3}\right\rceil$ of these $k$ vertices are in $\bar{V}$ since in each set $A_{j}$, we skip the three first vertices and then we pick one on three repetitively. If $t<k<2 t$ then the number of removed vertices is $q+\left\lceil\frac{k-t-2}{3}\right\rceil=\left\lceil\frac{k-2}{3}\right\rceil$ (since for the $t+1$ first vertices we remove $q$ of them and for the last $k-t-1$ vertices, we skip one and then we pick one on three repetitively, thus removing $\left\lceil\frac{k-t-2}{3}\right\rceil$ of them).

In both cases, we proved that $\bar{V}$ is a feedback set of cardinality at most $\left\lfloor\frac{n}{3}\right\rfloor=\left\lceil\frac{n-2}{3}\right\rceil$, which proves the proposition.

For the particular case $t=2$, the previous proposition gives a sharp result:

Corollary 1 For any $n \geq 3$,

$$
F\left(P_{n}(1,2)\right)=\left\lceil\frac{n-2}{3}\right\rceil .
$$

We shall now give an algorithm to find another feedback set for $P_{n}(1, t)$. This new set has cardinality closer to the optimal for specific values of $n$ compared to $t$.

Proposition 3 For any $t \geq 2$, and any $n>3 t$, let $t^{\prime}=2\left\lceil\frac{t}{2}\right\rceil$ and let $r$ be the integer such that $n \equiv$ $r \bmod 3 t^{\prime}, 0 \leq r<3 t^{\prime}$. Then

$$
F\left(P_{n}(1, t)\right) \leq \begin{cases}\frac{n-r}{3} & \text { if } 0 \leq r \leq t \\ \left\lfloor\frac{n}{3}-\frac{t}{6}\right\rfloor+3 & \text { if } t<r<3 t^{\prime}\end{cases}
$$

Proof: Let $n=3 t^{\prime} q+r$, let $G=P_{n}(1, t)$, let $G^{\prime}=P_{3 t^{\prime} q}(1, t)$ and let $G^{\prime \prime}=P_{r}(1, t)$.

The idea is to construct a periodic feedback set $\overline{V^{\prime}}$ of period $3 t^{\prime}$ for the $3 t^{\prime} q$ first vertices of $G$ (i.e. for $G^{\prime}$ ) and to use Proposition 1 to complete the FVS for the remaining $r$ vertices (i.e. for $G^{\prime \prime}$ ). We now distinguish two cases depending on the parity of $t$.

If $t$ is even then $t^{\prime}=t$. The FVS is constructed as follows: cut the set of $3 t$ consecutive vertices starting from vertex 0 in three blocks of size $t$, skip the first block, pick alternately one on two vertices of the second block and then again pick alternately one on two vertices of the last block, but starting from the second vertex of the block. Repeat the process for the next block of $3 t$ vertices, and so on. An illustration of this method is given in Part $a$ ) of Figure 5

Precisely, the FVS is defined by:

$$
\overline{V^{\prime}}=\bigcup_{j=0}^{q-1}\left\{\left\{3 t j+t+2 i, 0 \leq i \leq \frac{t-2}{2}\right\} \cup\left\{3 t j+2 t+2 i+1,0 \leq i \leq \frac{t-2}{2}\right\}\right\} .
$$




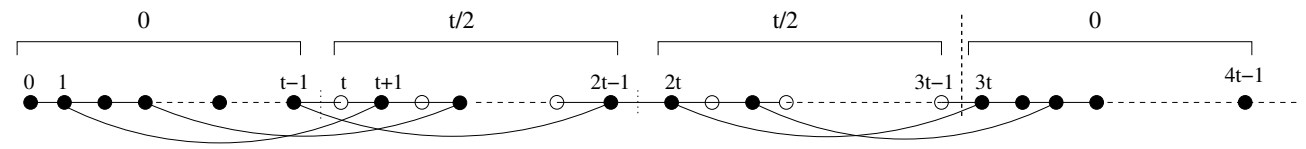

a)

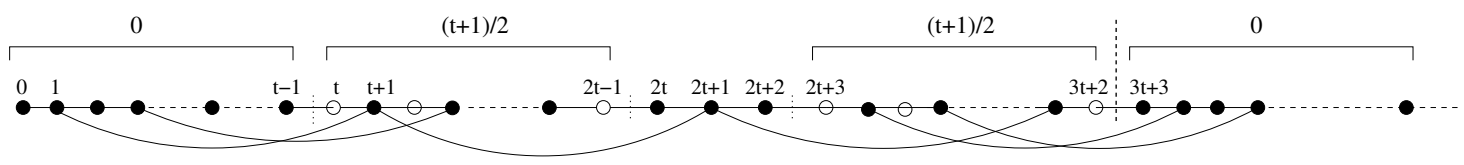

b)

Fig. 5: Structure of the FVS $\overline{V^{\prime}}$ for even $t$ on Part a) and for odd $t$ on Part b)

Hence, the cardinality of $\overline{V^{\prime}}$ satisfies $\left|\overline{V^{\prime}}\right|=2\left(\frac{t-2}{2}+1\right) q=t q=t^{\prime} q=\frac{n-r}{3}$. To see that $\overline{V^{\prime}}$ is a FVS, observe that in each block of $3 t$ vertices, the first block $B_{1}$ of $t$ vertices does not induce any cycle, and there is only one edge between the next block $B_{2}$ of $t$ vertices and the last block $B_{3}$ of $t$ vertices since the removed vertices are of different parity in $B_{2}$ and $B_{3}$. Thus, no cycle in $G \backslash \overline{V^{\prime}}$ can cross a block of $3 t$ vertices. Furthermore, as the vertices of the block $B_{3}$ are of degree one except the first vertex who has degree 2 , then they cannot form a cycle with the $t$ first vertices of the next block of $3 t$ vertices.

If $t$ is odd then $t^{\prime}=t+1$. The FVS is constructed as follows: take a block of $3 t^{\prime}=3 t+3$ consecutive vertices starting at vertex 0 , skip the $t$ first ones, pick alternately one on two of the next $t$ vertices, skip three vertices and then again pick alternately one on two of the last $t$ vertices. Repeat the process for the next block of $3 t^{\prime}$ vertices, and so on. An illustration of this method is given in Part $b$ ) of Figure 5

Precisely, the FVS is defined by:

$$
\overline{V^{\prime}}=\bigcup_{j=0}^{q-1}\left\{\left\{3 t^{\prime} j+t+2 i, 0 \leq i \leq \frac{t-1}{2}\right\} \cup\left\{3 t^{\prime} j+2 t+2 i+3,0 \leq i \leq \frac{t-1}{2}\right\}\right\} .
$$

Hence, the cardinality of $\overline{V^{\prime}}$ satisfies $\left|\overline{V^{\prime}}\right|=2\left(\frac{t-1}{2}+1\right) q=t^{\prime} q=\frac{n-r}{3}$. Among the three vertices between the first $2 t$ vertices (block $B_{1}$ and $B_{2}$ ) and the last $t$ vertices (block $B_{3}$ ) of any block of $3 t^{\prime}$ vertices, only one has degree 4 (but with two pendant edges incident to him) and the two other have degree 1. Moreover, removed vertices from the block $B_{2}$ and $B_{3}$ have the same parity. Thus, as the length of the chord is odd, there is no chordal edge between block $B_{2}$ and block $B_{3}$. Consequently, no cycle can cross a block of $3 t^{\prime}$ vertices.

For $0 \leq r \leq t, G^{\prime \prime}$ is a path and thus $\overline{V^{\prime \prime}}=\emptyset$ is a FVS. For $t<r \leq 3 t$, by Proposition 1 , there exists a FVS $\overline{V^{\prime \prime}}$ of $G^{\prime \prime}$. If $t$ is odd and $3 t+1 \leq r \leq 3 t^{\prime}-1=3 t+2$ then we add the one or two last vertices of $G^{\prime \prime}$ in the FVS $\overline{V^{\prime \prime}}$.

To see that the set $\bar{V}=\overline{V^{\prime}} \cup \overline{V^{\prime \prime}}$ is a FVS of $G$, it remains to show that no cycle is formed when making the union of $G^{\prime} \backslash \overline{V^{\prime}}$ and $G^{\prime \prime} \backslash \overline{V^{\prime \prime}}$. In fact, for both even and odd $t$, among the last $t-1$ vertices of $G^{\prime}$, the remaining vertices in $G^{\prime} \backslash \overline{V^{\prime}}$ are of degree 0 . Therefore, only one chordal edge will link a vertex of degree greater than 0 in $G^{\prime}$ to a vertex of $G^{\prime \prime}$ and no cycle can be formed. 
If $0 \leq r \leq t$ then $\bar{V}$ satisfies $|\bar{V}|=\frac{n-r}{3}$.

If $t<r \leq 2 t$ then $|\bar{V}|=\frac{n-r}{3}+\left\lceil\frac{r-t}{2}\right\rceil \leq \frac{2 n-2 r+3 r-3 t+6}{6} \leq \frac{n}{3}-\frac{t}{6}+1 \leq\left\lfloor\frac{n}{3}-\frac{t}{6}\right\rfloor+3$.

If $2 t<r<3 t^{\prime}$ then $|\bar{V}| \leq \frac{n-r}{3}+\left\lfloor\frac{r+2}{4}\right\rfloor+2 \leq \frac{4 n-4 r+3 r+6}{12}+2 \leq \frac{n}{3}-\frac{t}{6}+3$. As $|\bar{V}|$ is an integer, then $|\bar{V}| \leq\left\lfloor\frac{n}{3}-\frac{t}{6}\right\rfloor+3$.

Remark 1 The result of Proposition 3 is sharp when $n \equiv t \bmod 3 t^{\prime}$, with $t^{\prime}=2\left\lceil\frac{t}{2}\right\rceil$. We have then $F\left(P_{n}(1, t)\right)=\frac{n-t}{3}$.

\section{$2.2 D=\{1, s, t\}$}

Proposition 4 For any integers $n$, $s$ and $t$, with $t \leq n-1$ and $2 \leq s<t$,

$$
\left\lceil\frac{2 n-s-t}{5}\right\rceil \leq F\left(P_{n}(1, s, t)\right) \leq\left\lceil\frac{n}{2}\right\rceil .
$$

Proof: Let $G=P_{n}(1, s, t)$. Notice that, for any $d \in D$, there are exactly $n-d$ chords of length $d$ in $P_{n}(D)$. Thus the lower bound comes from Lemma 1 as $G$ is of order $n$, size $3 n-s-t-1$ and maximum degree 6 .

To prove the upper bound we will discuss two cases. In each one, we remove a vertex set from $G$ which generates a feedback.

Case 1: $s$ or $t$ is odd. Let $\bar{V}=\left\{2 j+1,0 \leq j \leq\left\lfloor\frac{n-2}{2}\right\rfloor\right\}$ be the vertex set which we remove from $G$. As a chord of odd length always joins two vertices of different parity and as we remove all vertices of odd index, then the subgraph induced by $V \backslash \bar{V}$ is either an independent set (if both $s$ and $t$ are odd) or a disjoint union of paths. So $\bar{V}$ is a FVS and $|\bar{V}|=\left\lfloor\frac{n}{2}\right\rfloor$.

Case 2: $s$ and $t$ are even. For each $k, 1 \leq k \leq\left\lceil\frac{n}{t}\right\rceil$, if $k$ is even we remove from $G$ the set $\bar{V}_{k}=\{u=$ $\left.2 j+1+(k-1) t, u \leq n-1,0 \leq j \leq \frac{t-2}{2}\right\}$, otherwise we remove $\bar{V}_{k}=\{u=2 j+(k-1) t, u \leq$ $\left.n-1,0 \leq j \leq \frac{t-2}{2}\right\}$. See Figure 6 for an illustration when $s=4$ and $t=6$.

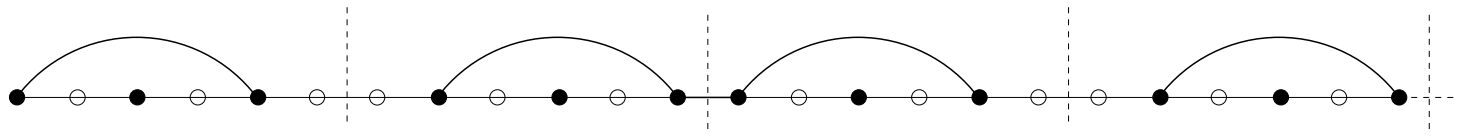

Fig. 6: A FVS of $P_{n}(1,4,6)$.

One can see that, as $s$ and $t$ are even, the chords of length $s$ or $t$ of $G$ always join vertices of the same parity. Moreover, as we remove alternately odd and even vertices by blocks of $t$ vertices, all chords of length $t$ are removed and all chords of length $s$ between two consecutive blocks of $t$ vertices are removed too. Thus in each block we obtain a forest and since chords of length one are all removed except those between some consecutive blocks, the set $\bar{V}=\bigcup_{k} \bar{V}_{k}$ is a FVS.

Notice that we have $\left|\bar{V}_{k}\right|=\frac{t}{2}$ and $|\bar{V}| \leq\left\lceil\frac{n}{2}\right\rceil$ since in each block of $t$ vertices, we alternately remove one vertex on two starting from the first or the second vertex of the block. 
Proposition 5 For any integer $n \geq 4$

$$
4\left\lfloor\frac{n}{8}\right\rfloor \leq F\left(P_{n}(1,2,4)\right) \leq\left\lceil\frac{n}{2}\right\rceil
$$

Proof: To prove the lower bound, let $\bar{V}$ be any FVS of $G=P_{n}(1,2,4)$. Consider the first 6 vertices of $G$. Note that three consecutive vertices in $G$ form a cycle. Thus we have two cases to study: if only two vertices over the six are in $\bar{V}$, then the only possibility is to remove vertices 1 and 4 (see Figure 7). But in this case, the two following vertices (6 and 7) must be in $\bar{V}$, otherwise a cycle is produced. Then, among the 8 first vertices of $G, 4$ of them are in $\bar{V}$. If three or more vertices among the first 6 one are in $\bar{V}$ then half or more than half of the vertices are removed on this part of $G$. Therefore, on the first six or eight vertices of $G$, at least half of them are in $\bar{V}$. Next, iterating this process on the six next vertices of $G$ and so on until the end of the graph, we obtain the desired lower bound.
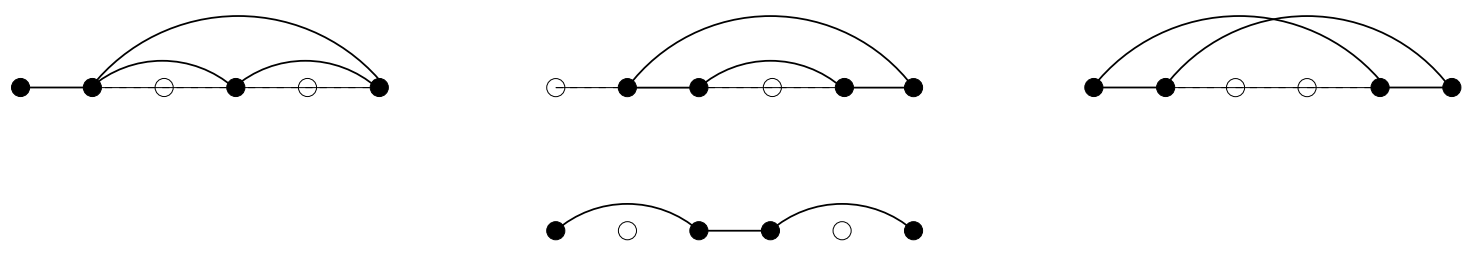

Fig. 7: The graph $P_{6}(1,2,4)$ with two vertices removed. The three configurations on the top of the figure produce a cycle.

The upper bound follows from Proposition 4

$2.3 D=\{1,2,3, \ldots, t\}$

Proposition 6 For any $n \geq 5$ and $t \leq \frac{n-1}{2}$,

$$
F\left(P_{n}(\{1,2,3, \ldots, t\})\right)= \begin{cases}\left\lfloor\frac{n}{t+1}\right\rfloor(t-1) & \text { if } n \equiv 0,1 \bmod (t+1), \\ n-2\left\lfloor\frac{n}{t+1}\right\rfloor-2 & \text { otherwise }\end{cases}
$$

Proof: Let $G=P_{n}(\{1,2, \ldots, t\})$ and let $n=p(t+1)+q$, with $0 \leq q<t+1$ and $p \geq 1$.

The subgraphs $G_{i}, 0 \leq i \leq p-1$ of $G$ induced by vertices $\{(t+1) i+j, 0 \leq j \leq t\}$ and $G_{p}$ induced by vertices $\{p(t+1)+j, 0 \leq j \leq q-1\}$ are cliques. So any FVS of $G$ must contain at least $t-1$ vertices of each $G_{i}, 0 \leq i \leq p-1$ and at least $q-2$ vertices of $G_{p}$ if $q \geq 2$. Thus, if $q \leq 1$ then $F(G) \geq$ $p(t-1)=\left\lfloor\frac{n}{t+1}\right\rfloor(t-1)$, otherwise, $F(G) \geq p(t-1)+q-2=p(t+1)+q-2 p-2=n-2\left\lfloor\frac{n}{t+1}\right\rfloor-2$.

To show the equality, we give a FVS with the desired number of vertices (see Figure 8): $\bar{V}=\{i+$ $k(t+1), 2 \leq i \leq t, 0 \leq k \leq p-1\} \cup\{p(t+1)+i, 2 \leq i \leq q-1\}$.

By construction, there is only one chord between two consecutive blocks of $(t+1)$ vertices (a chord of length $t$ between the second vertex of the block and the first vertex of the next block). Thus the graph $G \backslash \bar{V}$ is a path. Moreover, we have $|\bar{V}|=p(t-1)$ if $q \leq 1$ and $|\bar{V}|=p(t-1)+q-2$ if $q \geq 2$. 


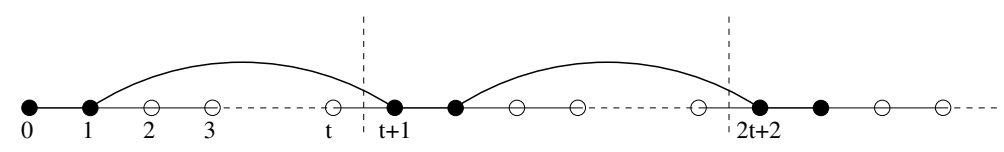

Fig. 8: A FVS of $P_{n}(1,2,3, \ldots, t)$

Remark 2 For the general distance graph $F\left(P_{n}\left(d_{1}, d_{2}, \ldots, d_{t}\right)\right)$ and for any $n \geq 5$ and $1 \leq d_{1}<$ $d_{2}<\ldots<d_{t}$, with $d_{t} \leq n-1$ and at most one $d_{i}$ even, we have that $F\left(P_{n}\left(d_{1}, d_{2}, \ldots, d_{t}\right)\right) \leq\left\lfloor\frac{n}{2}\right\rfloor$. Effectively, if we remember that a chord of odd length always joins vertices of different parity, we can see that the set $\bar{V}=\left\{2 j+1,0 \leq j \leq \frac{n-2}{2}\right\}$ is a feedback vertex set.

\section{Feedback vertex sets of circulant graphs}

In this section, we shall always assume that $t \leq \frac{n}{2}$.

Proposition 7 Let $n$ be an integer and let $D \subset \mathbb{Z}_{n}$ and $m \geq \max _{d \in D}\{d\}$. Then

$$
F\left(P_{n}(D)\right) \leq F\left(C_{n}(D)\right) \leq F\left(P_{n-m}(D)\right)+m .
$$

Proof: The lower bound is trivial as $P_{n}(D)$ is a partial subgraph of $C_{n}(D)$. For the upper bound, if we cut $m$ consecutive vertices of $C_{n}(D)$, we obtain a graph isomorphic to $P_{n-m}(D)$. As $m \geq \max _{d \in D}\{d\}$, no chord can cross this block of $m$ vertices. Thus it is sufficient to add these $m$ vertices to a MFVS of $P_{n-m}(D)$ to obtain a FVS of the circulant graph $C_{n}(D)$.

\section{1 $D=\{1, t\}$}

Proposition 8 For any $n \geq 5$ and $t<\frac{n}{2}$,

$$
\left\lceil\frac{n+1}{3}\right\rceil \leq F\left(C_{n}(1, t)\right) \leq \begin{cases}\left\lceil\frac{n+t}{3}\right\rceil+1 & \text { ift } \equiv 0 \bmod 3 \\ \left\lceil\frac{n+t}{3}\right\rceil & \text { ift } \neq \equiv 0 \bmod 3 .\end{cases}
$$

Proof: Let $G=C_{n}(1, t)$. The lower bound comes from Lemma 1 as $G$ is of order $n$, size $2 n$ and maximum degree 4 . In order to prove the upper bound, we consider the circulant graph as a distance graph plus some vertices and edges: Let $B$ be the subgraph of $G$ induced by vertices $t, t+1, \ldots, n-1$. $B$ is the distance graph $P_{n-t}(1, t)$ and since $n-t>t$, by Proposition 2 there exists a FVS $\bar{V}_{B}$ of $B$ of size $\left\lceil\frac{n-t-2}{3}\right\rceil$. A FVS $\bar{V}_{G}$ for $G$ can be constructed by adding to $\bar{V}_{B}$ a subset $N$ of the remaining $t$ vertices $0,1, \ldots, t-1: N=\left\{j, 0 \leq j \leq t-1,(j+t) \notin \bar{V}_{B}\right\}$. Then $\bar{V}_{G}=\bar{V}_{B} \cup N$.

$B \backslash \bar{V}_{B}$ induces a forest in $G$ and by the choice of $N$, there is no edge between a vertex of the first block of $t$ vertices and a vertex of the second block of $t$ vertices. Moreover, as it can be seen in the proof of Proposition 2, no two consecutive vertices of $P_{n-t}(1, t)$ are in $\bar{V}_{B}$. Thus, among the $t$ first vertices of $G$, at least one of any two consecutive vertices is in $N$. Consequently, there is no possibility to form a cycle since the $t$ first vertices of $G$ become of degree at most one. 
Moreover, by the construction of $\bar{V}_{B}$ (see proof of Proposition 2), if $t \not \equiv 0 \bmod 3$ then we have $|N|=t-\left\lceil\frac{t-2}{3}\right\rceil=\left\lceil\frac{2 t}{3}\right\rceil$. Thus $\left|\bar{V}_{G}\right| \leq\left\lceil\frac{n-t-2}{3}\right\rceil+\left\lceil\frac{2 t}{3}\right\rceil \leq\left\lceil\frac{n+t}{3}\right\rceil$. If $t \equiv 0 \bmod 3$ then $|N|=$ $t-\left\lceil\frac{t-3}{3}\right\rceil=\left\lceil\frac{2 t}{3}\right\rceil+1$. Thus $\left|\bar{V}_{G}\right| \leq\left\lceil\frac{n+t}{3}\right\rceil+1$.

For the special case $t=2$, the previous proposition gives a quasi optimal result. The exact value is determined in the following proposition:

Proposition 9 For any $n \geq 4$, we have

$$
F\left(C_{n}(1,2)\right)=\left\lceil\frac{n+2}{3}\right\rceil .
$$

Proof: By Lemma 1, $F\left(C_{n}(1,2)\right) \geq\left\lceil\frac{n+1}{3}\right\rceil$. Observe that in case $n=3 k+1$ or $3 k$, we have $\left\lceil\frac{n+1}{3}\right\rceil=$ $\left\lceil\frac{n+2}{3}\right\rceil$, thus Proposition 8 gives the result. So let us study the case where $n=3 k+2$. The proof is done by contradiction. Assume that $F\left(C_{n}(1,2)\right) \leq\left\lceil\frac{n+2}{3}\right\rceil-1$, i.e. $F\left(C_{n}(1,2)\right) \leq k+1$. Then there exists a subset of vertices, say $S$, such that the induced subgraph $G \backslash S$ is a forest. Give a clockwise orientation to the cycle $C=C_{n}(1)$. Let $S=\left\{s_{0}, s_{1}, \ldots, s_{q-1}\right\}$, with $1 \leq q \leq k+1$ such that these vertices hold in this order on $C$. Let $s_{i}^{+}$( $s_{i}^{-}$, respectively) be the successor (predecessor, respectively) of vertex $s_{i}$ in $C_{n}$ with respect to the orientation (indices are taken modulo $q$ ).

Denote by $C_{i}$ the part of cycle given by the segment $\left(s_{i}^{+} C s_{i+1}^{-}\right)$. Suppose first that there exists $0 \leq$ $j \leq q-1$, such that the edge $s_{j} s_{j+1} \in E\left(C_{n}\right)$, i.e. such that $\left|C_{j}\right|=0$. Let $P_{n-2}(1,2)$ be given by $C_{n}(1,2) \backslash\left\{s_{j}, s_{j+1}\right\}$. By Proposition 2, $F\left(P_{n-2}(1,2)\right)=\left\lceil\frac{n-4}{3}\right\rceil=k$. So $F\left(C_{n}(1,2)\right) \geq k+2$, a contradiction.

Now suppose that for all $0 \leq i \leq q-1,\left|C_{i}\right|>0$. Observe that there does not exist $C_{j}$, with $0 \leq j \leq q-1$, such that $\left|C_{j}\right| \geq 3$, otherwise we obtain a cycle of size 3 . We deduce that for each $i, 0 \leq i \leq q-1$, we have $1 \leq\left|C_{i}\right| \leq 2$. It means that it is possible, in this case, to construct a cycle which passes by each $C_{i}$ by taking all vertices of each segment: let $x_{i}$ and $y_{i}$ be the vertices of $C_{i}$, where $x_{i}=y_{i}$ if $\left|C_{i}\right|=1$ and $y_{i}=x_{i}^{+}$if $\left|C_{i}\right|=2$. Then the cycle is given by $\left(x_{0}, y_{0}, x_{1}, y_{1}, \ldots, x_{q-1}, y_{q-1}, x_{0}\right)$. Therefore $G \backslash S$ is not a forest, a contradiction. Then any MFVS has cardinality at least $k+2=\left\lceil\frac{n+2}{3}\right\rceil$. Thus, Proposition 8 gives $F\left(C_{n}(1,2)\right)=\left\lceil\frac{n+2}{3}\right\rceil$.

If $n=2 p$ is even and $t=\frac{n}{2}=p$, then the circulant graph $C_{2 p}(1, p)$ is cubic. In that case, it is easy to compute the cardinality of a MFVS.

Proposition 10 for any $p \geq 2$,

$$
F\left(C_{2 p}(1, p)\right)=\left\lceil\frac{p+1}{2}\right\rceil .
$$

Proof: By Lemma $1, F\left(C_{2 p}(1, p)\right) \geq\left\lceil\frac{p+1}{2}\right\rceil$.

If $p$ is even, then the set $\bar{V}=\{0\} \cup\left\{2 j+1,0 \leq j \leq \frac{p}{2}-1\right\}$ is a FVS of cardinality $\frac{p}{2}+1=\left\lceil\frac{p+1}{2}\right\rceil$. If $p$ is odd, then the set $\bar{V}=\left\{2 j, 0 \leq j \leq \frac{p-1}{2}\right\}$ is a FVS of cardinality $\frac{p-1}{2}+1=\left\lceil\frac{p+1}{2}\right\rceil$. 
3.2 $D=\{1, s, t\}$

Proposition 11 For any integers $n, s$ and $t$, with $t \leq \frac{n-1}{2}$ and $2 \leq s<t$,

$$
\left\lceil\frac{2 n+1}{5}\right\rceil \leq F\left(C_{n}(1, s, t)\right) \leq\left\lceil\frac{n+t}{2}\right\rceil .
$$

Proof: This proposition immediately follows from Lemma 1. Proposition 4 and Proposition 7

Corollary 2 For any integer $n \geq 9$,

$$
4\left\lfloor\frac{n}{8}\right\rfloor \leq F\left(C_{n}(1,2,4)\right) \leq\left\lceil\frac{n+4}{2}\right\rceil .
$$

Proof: The lower bound follows from Proposition 5 as $P_{n}(1,2,4)$ is a subgraph of $C_{n}(1,2,4)$. The upper bound is given by Proposition 11 with $m=t=4$.

\section{3 $D=\{1,2, \ldots, t\}$}

Proposition 12 For any $n \geq 5$ and $3 \leq t \leq \frac{n-1}{2}$, let $p$ and $q$ be integers such that $n=p(t+1)+q$, $q<t+1$. Then

$$
\begin{gathered}
F\left(C_{n}(1,2, \ldots, t)\right)=n-2 p+1, \text { if } q=0 ; \\
n-2 p-1 \leq F\left(C_{n}(1,2, \ldots, t)\right) \leq n-2 p, \text { if } q \geq 1 .
\end{gathered}
$$

Proof: Let $G=C_{n}(1,2, \ldots, t)$ and let $n=(t+1) p+q$, with $q<t+1$. We cut up the vertices of $G$ successively into $p+1$ sets of vertices, in other words we have

$B_{i}=\{(i(t+1), i(t+1)+1, \ldots,(i+1)(t+1)-1\}$, for $0 \leq i \leq p-1$, and

$B_{p}=\{p(t+1), p(t+1)+1, \ldots, p(t+1)+q-1\}$.

As $\left|B_{i}\right| \leq t+1$, any subgraph of $G$ induced by $B_{i}$, for $0 \leq i \leq p$, is a clique. Thus any triplet of vertices of this subgraph forms a cycle. So a FVS in this case must contain at least $t-1$ vertices of each set $B_{i}$, for $0 \leq i \leq p-1$ and $q-2$ vertices of $B_{p}$ if $q \geq 2$.

However, there is still a "big" cycle since if it remains two vertices in each block $B_{i}$ (one vertex in $B_{p}$ if $q=1$ ), then there is at least an edge between any two successive blocks. Thus any FVS must contain an extra vertex.

Consequently, if $q=0$ then we have $F\left(C_{n}(1,2, \ldots, t)\right) \geq p(t-1)+1=n-2 p+1$ and if $q \geq 1$, then $F\left(C_{n}(1,2, \ldots, t)\right) \geq p(t-1)+\max (q-2,1)+1 \geq p(t+1)+q-2 p-1=n-2 p-1$.

We construct a FVS $\bar{V}$ by taking the $t-1$ first vertices of each $B_{i}$, with $0 \leq i \leq p-1$, and the $q$ vertices of $B_{p}$. By construction, there is only one chord between two consecutive blocks (the chord of length $t$ joining the last vertex of the first block with the last but one vertex of the next block). If $q \neq 0$ then $G \backslash \bar{V}$ is the path $P=(t-1, t, 2 t, 2 t+1,3 t+1,3 t+2, \ldots, p(t+1)-2, p(t+1)-1)$. Otherwise, if $q=0$ then $G \backslash \bar{V}$ consists in the cycle $C=P \cup\{p(t+1)-1, t-1\}$. In this case, we add any vertex of the cycle $C$ to $\bar{V}$.

Observe that if $q=0$ then $|\bar{V}| \leq p(t-1)+1=n-2 p+1$ and if $q \geq 1$ then $|\bar{V}| \leq p(t-1)+q=n-2 p$. Hence we obtain the desired result. 
Remark 3 From Proposition 7 and Remark 2 the following can be easily obtained: for any $n \geq 5$ and $1 \leq d_{1}<d_{2}<\ldots<d_{t}$, with $d_{t} \leq \frac{n-1}{2}$,

i) if $n$ is even and if all $d_{i}$ are odd, then $F\left(C_{n}\left(d_{1}, d_{2}, \ldots, d_{t}\right)\right) \leq\left\lfloor\frac{n}{2}\right\rfloor$,

ii) if $n$ is odd and if all $d_{i}$ are odd, then $F\left(C_{n}\left(d_{1}, d_{2}, \ldots, d_{t}\right)\right) \leq\left\lfloor\frac{n+d_{t}}{2}\right\rfloor$,

iii) if $n$ is even and if there exists only one even $d_{i}$, with $1 \leq i \leq t$, then $F\left(C_{n}\left(d_{1}, d_{2}, \ldots, d_{t}\right)\right) \leq\left\lfloor\frac{n+d_{i}}{2}\right\rfloor$.

\section{Conclusion}

We have derived several bounds for the cardinality of a minimum feedback vertex set of distance and circulant graphs. The approximation ratios of our algorithms are in many cases equal or asymptotically close to one for circulant graphs and for distance graphs with chords of length at most half the number of vertices.

For isomorphism reasons, our results concerning circulant graphs defined by the set $D=\{1, t\}$ can also be applied to many other circulants of degree 4 . For instance, the following isomorphism is easy to observe: if $\operatorname{gcd}\left(d_{1}, n\right)=1$ or $\operatorname{gcd}\left(d_{2}, n\right)=1$ then there exists $t$ such that $C_{n}\left(d_{1}, d_{2}\right) \equiv C_{n}(1, t)$.

\section{Acknowledgements}

We wish to thank the anonymous referees for helpful comments that allow to improve the paper. 


\section{References}

[BBF99] V. Bafna, P. Berman, and T. Fujito. A 2-approximation algorithm for the undirected feedback vertex set problem. SIAM J. Discrete Math., 12(3):289-297, 1999.

[BG94] A. Becker and D. Geiger. Approximation algorithms for the loop cutset problem. In Proc. of the 10th Conference on Uncertainty in Artificial Intelligence, pages 60-68, 1994.

[BCH95] J.-C. Bermond, F. Comellas and D. F. Hsu. Distributed Loop Computer Networks: A Survey J. of Parallel and Distributed Computing, 24:2-10, 1995.

[CKK02] I. Caragiannis, C. Kaklamanis, and P. Kanellopoulos. New bounds on the size of the minimum feedback vertex set in meshes and butterflies. Inform. Process. Lett., 83(5):275-280, 2002.

[EES85] R.B. Eggleton, P. Erdös, and D.K. Skilton. Coloring the real line. Journal of Combinatorial Theory, B39:86-100, 1985.

[EES90] R. B. Eggleton, P. Erdős, and D. K. Skilton. Colouring prime distance graphs. Graphs Combin., 6(1):17-32, 1990.

[FGR02] G. Fertin, E. Godard, and A. Raspaud. Minimum feedback vertex set and acyclic coloring. Inform. Process. Lett., 84(3):131-139, 2002.

[FLP00] R. Focardi, F. L. Luccio, and D. Peleg. Feedback vertex set in hypercubes. Inform. Process. Lett., 76(1-2):1-5, 2000.

[FPR99] P. Festa, P.M. Pardalos, and M.G. Resende. Feedback set problem. In Handbook of Combinatorial Optimization, volume 4, pages 209-258. Kluwer Academic, 1999.

[GJ79] M. R. Garey and D. S. Johnson. Computers and intractability. W. H. Freeman and Co., San Francisco, Calif., 1979.

[EK03] B. Effantin and H. Kheddouci. The b-chromatic number of some power graphs. Discrete Mathematics and Theoretical Computer Science, 6(1):45-54, 2003.

[Luc98] F. L. Luccio. Almost exact minimum feedback vertex set in meshes and butterflies. Inform. Process. Lett., 66(2):59-64, 1998.

[Luc03] F. L. Luccio. Minimum Feedback Vertex Set in Pyramid and Mesh of Trees Networks. In Proc. of the 10th Colloquium on Structural Information and Communication Complexity (SIROCCO'03), June 2003, Umea, Sweden.

[Pik03] D. A. Pike. Decycling hypercubes. Graphs and Combinatorics, 19(4): 547-550, 2003.

[PK05] D. A. Pike and Y. Zou. Decycling Cartesian products of two cycles. SIAM Journal on Discrete Mathematics, 19(3): 651-663, 2005.

[Tog00] O. Togni. Placement de convertisseurs de longueur d'onde dans les réseaux optiques. In Proc. of 2ièmes Rencontres Francophones sur les Aspects Algorithmiques des Télécommunications (Algotel2000), pages 35-40. INRIA, 2000.

[Voi99] M. Voigt. Colouring of distance graphs. Ars Combin., 52:3-12, 1999. 